\title{
LA INTERPRETACIÓN DE LAS NORMAS TRIBUTARIAS EN FUNCIÓN A LA REALIDAD DE LOS HECHOS ECONÓMICOS
}

Abog. Lourdes Calderón Aguilar*

\section{RESUMEN E INTRODUCCIÓN}

El tema que nos ocupa es uno que ha sido tratado largamente por destacados tributaristas del medio $\mathrm{y}$, básicamente, supone establecer, que en las relaciones juridicas, como la tributaria, la primacía de los hechos contables, no puede ser dejada de observar. Las interminables contingencias entre la norma legal y la contable quedan así sometidas a los criterios resolutivos de los altos tribunales, quienes deberán, antes que a la forma, dar primacía a los hechos económicos que las recubren.

\section{Antecedentes Históricos}

El enfoque económico en la interpretación de las normas tributarias surgió en Alemania en el año 1919, incorporado en la Ordenanza Tributaria del Reitch, $u$ Ordenamiento Tributario del Reitch, ante la imposibilidad de los tribunales fiscales de aplicar los gravámenes, ya que la base de la imposición estaba establecida por elementos del derecho civil.

Su principal propulsor, Enno Becker, inspiró la fórmula del artículo $4^{\circ}$ del Ordenamiento, por la que se postuló que en la interpretación de las leyes tributarias debe tenerse en cuenta su finalidad, su significado y el desarrollo de las circunstancias.

Posteriormente, este principio fue recogido en Francia en la década de los 20 por medio de su formulación por Trotabas, así como en Italia y Suiza, siendo Argentina el primer país latinoamericano en darle vigencia legal, con las modificaciones que se realizaron a la ley 11.683 en el año 1947, así como con el Código Fiscal de Buenos Aires de 1948, cuyo principal inspirador fue Dino Jarach. Siguiendo estos antecedentes, luego fue incorporado al Proyecto de Código Fiscal de Uruguay en 1959.

Un año antes, esto es en 1958 , el principio fue admitido en la II Jornada Latinoamericana de Derecho Tributario aunque de manera escueta, aplicándose sólo cuando así correspondiera a la naturaleza de los hechos gravados.

En 1967, el Modelo de Código Tributario para América Latina lo acoge en los siguientes términos:

"Artículo 8. Cuando la norma relativa al hecho generador se refiera a situaciones definidas por otras ramas jurídicas, sin remitirse ni apartarse expresamente de ellas, el intérprete puede asignarle el significado que más se adapte a la

* Docente Asociada de la Facultad de Ciencias Contables 
realidad considerada por la ley al crear el tributo.

Las formas juridicas adoptadas por los contribuyentes no obligan al intérprete, quien podrá atribuir a las situaciones y actos ocurridos una significación acorde con los hechos, cuando de la ley tributaria surja que el hecho generador fue definido atendiendo a la realidad y no a la forma juridica.

Cuando las formas jurídicas sean manifiestamente inapropiadas a la realidad de los hechos gravados $y$ ello se traduzca en una disminución de la cuantía de las obligaciones, la ley tributaria se aplicará prescindiendo de tales formas.

La exposición de motivos, dice que el proyecto parte de la base de que el intérprete tiene que adjudicar a la norma su verdadero significado, para lo cual no puede estar subordinado a definiciones estructuradas sobre fundamentos y con finalidades distintas que pueden o no coincidir con los de las normas tributarias.

A raíz de su incorporación en el Modelo, otras legislaciones tributarias lo recogieron indistintamente; en algunos casos parcialmente, como en Bolivia, que solamente tomó en cuenta el segundo párrafo, y en otros completamente, como es el caso de Costa Rica, que lo reprodujo textualmente. Ecuador, por su parte, reguló de manera diferente el problema del primer párrafo, estableciendo que las disposiciones de otras ramas se aplicarian únicamente como normas supletorias. Paraguay y Uruguay incluyeron en las disposiciones de aplicación general, en 1992, los dos primeros párrafos, y recientemente hizo lo mismo el Perú con el Decreto Legislativo 816, modificado por la Ley 26663.

\section{Fundamentos del principio de la primacia de la realidad}

De acuerdo con el maestro Giuliani Founrouge, el fundamento del principio estriba en atender al contenido económico real de las circunstancias comprendidas, prescindiendo de las formas y estructuras inadecuadas. En otras palabras, refiere, que si los contribuyentes, por ignorancia, error o mala fe, cubren o exteriorizan sus propósitos efectivos, mediante apariencias formales distintas (por ejemplo encubren una compra venta bajo el aspecto de una donación), el fisco puede prescindir de esas apariencias y determinar la obligación tributaria según la realidad oculta, sin necesidad de demostrar la nulidad del acto jurídico aparente o accionar por la vía de la simulación.

El sustento de tal apreciación está en la calidad de Orden Público que ostentan las normas tributarias, por lo cual han de aplicarse tratando de conservar la situación de armonia y estabilidad social que existe en razón del acatamiento que todos los individuos por igual deben a las instituciones, cuando el Estado exige el cumplimiento de sus reglas con carácter de necesidad $y$ de irrenunciabilidad.

Para Héctor Villegas, la interpretación según la realidad económica, se basa en que el criterio para distribuir la carga tributaria surge de una valoración política de la capacidad contributiva que el legislador efectuó teniendo en cuenta 
precisamente la realidad económica. Siendo ello así, sólo se logrará descubrir el verdadero sentido $y$ alcance de la ley recurriendo a esa misma realidad económica que se quiso aprehender en el momento genésico del tributo".

Para Araujo Falcao, el fundamento del método (para nosotros en mucho coincidente con Founrouge) estriba en el derecho que tiene el Estado a percibir los tributos de acuerdo con la ley (normas pues de orden público) en situaciones de evasión fiscal; esto es, cuando existe una distorsión de la forma jurídica o una atipicidad o anormalidad de ésta frente a la relación económica que se exterioriza mediante aquélla. Si se configura esta hipótesis, las formas juridicas adoptadas deben ser ajustadas a la realidad, efectuando las correcciones a las liquidaciones, o prescindiendo de estas formas, incluso de la personeria juridica.

Como bien explica Julio Fernández, para los que propugnan esta teoria, en todo acto jurídico existe una intención jurídica o intentio juris, y una intención empírica o intentio factis. $\mathrm{El}$ mismo autor, citando a Dino Jarach, sostiene que en toda relación juridica de derecho civil y comercial deben diferenciarse en la voluntad de las partes dos aspectos, una voluntad empírica la de realizar un negocio, una operación económica, y una voluntad juridica, que es la de que el negocio encuadre dentro de un determinado molde o figura del derecho civil y comercial. Como sostiene Villegas, la intención empírica es la que se encamina a obtener el resultado económico para cuyo fin el acto se realiza, mientras que la intención jurídica será aquélla que se dirige a alcanzar, mediante el acto o negocio, determinados efectos juridicos asi deseados por las partes, quienes escogerán la forma mediante la cual sientan más protegidos sus derechos o mediante la que les sea más fácil conseguir el cumplimiento de la otra o mediante la cual paguen menos tributos.

Siendo ello así, la interpretación según la realidad económica supondria, en buena cuenta, desconocer la eficacia de los efectos jurídicos, si las partes han distorsionado la realidad de la operación económica utilizando una forma jurídica inapropiada que se busca al sólo efecto de defraudar al fisco.

Para algunos entendidos, el principio de la primacía de la realidad no es sino la sanción del Estado a la evasión fiscal cuando el sujeto pasivo pretende soslayar la obligación tributaria mediante el fraude. Veremos más adelante que tal no es la verdadera noción.

\section{Diferencia entre el principio de la primacia de la realidad de los hechos económicos con la teoria del disregard o doctrina de la penetración}

La idea de penetrar en el ropaje jurídico para hurgar en la realidad de los hechos, ha tenido aplicación en materia de sociedades mercantiles, dando lugar a decisiones judiciales y obras de doctrina que han logrado gran repercusión. Esa interpretación realista ha tenido exteriorización en los Estados Unidos, con el nombre de la teoria del "disregard of legal entity", denominación que en Francia y en Suiza se conoce como la transparencia fiscal, que considera a la persona jurídica como 
algo transparente, y que permite prescindir de ella como sujeto de obligaciones tributarias para incidir sobre las personas físicas que las integran. Además, surte sus efectos en materia de sociedades vinculadas o imbricadas cuando la apariencia juridica oculta subordinación económica o financiera, lo que se conoce en Alemania como la teoria del órgano, segūn la cual, cuando existe independencia financiera, organizativa o económica, se considera que la entidad dominada es un órgano de la dominante, con la cual se integra en un solo ente.

En algunas oportunidades, se ha considerado que el principio de la realidad económica es coincidente con el de disregard o la teoría de la penetración.

Sin embargo, como bien aprecia Valdés Costa, sin perjuicio de reconocer ciertos aspectos comunes, se presentan diferencias sustanciales.

Así, refiere que de común tienen los medios, o sea la facultad, del intérprete de desconocer la eficacia de la forma juridica para adaptarla a la realidad. Es decir, la predominancia de la substancia sobre la forma. Pero, como diferencias pueden anotarse:

1. El disregard procede en caso de fraude, mientras que el principio tributario de la primacía de la realidad de los hechos económicos, en todos los casos en que la forma no se ajuste a la realidad considerada en la ley, se trate de fraude o no.

2. El disregard se aplica en perjuicio de quien utilizó la forma; mientras que el principio en su perjuicio o beneficio.
3. El disregard implica una simulación de actos jurídicos, una desfiguración de los hechos; mientras que en el principio de primacía de la realidad de los hechos económicos, la forma puede coincidir con la realidad desde el punto de vista del derecho privado, pero no con el contenido, fundamento o finalidad de la ley tributaria.

4. El disregard o legal entity, como su nombre lo indica, consiste en el desconocimiento de la personería jurídica; mientras que, para el principio tributario, existen otras posibilidades.

La diferencia sustancial, pues, es que el disregard se limita expresamente a los casos en que existe fraude o abuso, con lo cual se elimina la posibilidad de que el contribuyente pueda aplicar el principio de la realidad en su favor, como sí procede, por ejemplo,en el caso uruguayo, que sostiene esto como un aspecto esencial de su doctrina.

Precisamente, la jurisprudencia rioplatense fue notoriamente innovadora en el tratamiento del tema, en la resoluciòn de casos que tuvieron grandes repercusiones.

Se tiene así el famoso caso de la "Parke Davis, "en el cual la Corte Suprema Argentina estableció que dos sociedades, una local y otra extranjera, que se diferenciaban desde el punto de vista del derecho privado pero que integraban una sola unidad económica, debian ser consideradas impositivamente como una sola entidad, por lo cual el pago de aparentes regalías no era más que un retiro disimulado de utilidades sujetas al impuesto a las 
ganancias, por cuanto todo contrato supone la existencia de dos partes y nadie puede contratar consigo mismo.

Otro caso importante estuvo referido a la empresa "Mellor Goldwin en el cual la Corte Suprema de Argentina adoptó el criterio de descorrer el velo societario y por aplicación de la doctrina de la penetración estableció que una sociedad casi integramente dominada por otra no autoriza a reconocer la existencia de convenciones entre ellas, por cuanto debe prevalecer la razón del derecho sobre el ritualismo juridico formal, aprehendiendo la realidad juridica objetiva, y ella indica que tampoco en esta situación son aceptables operaciones que aparentemente significan el traslado de mercaderias entre ambos elementos de un mismo conjunto económico.

Asimismo, puede citarse el caso de la empresa "Ford Motor de Argentina", en el cual la misma Corte Suprema de Argentina resolvió que si la exportadora del exterior y la importadora local se hallaban en la situación de matriz $y$ filial, integrando un conjunto económico, las sumas pagadas en concepto de intereses por operaciones de pago diferido no revisten ese carácter para el impuesto a los réditos, sino el de un pago o remesa de utilidades de la entidad local en beneficio de la extranjera, aclarando que en hipótesis de conjunto económico o situaciones equivalentes, las prestaciones y contraprestaciones deben ajustarse a los principios del aporte y de la utilidad.

En el Uruguay, por su parte, la sentencia del Tribunal de lo contencioso administrativo, Número 17 de 1977, prescindió de la consideración de la persona jurídica de derecho privado. El caso se refería a una empresa de transporte colectivo de Montevideo constituida en forma de sociedad anónima, cuyos accionistas eran exclusivamente propietarios de las unidades de transporte y que funcionaba exclusivamente para ellos sin obtener beneficios. En este caso, el reconocimiento judicial de que la realidad económica prevalece sobre las formas juridicas del derecho privado fue reclamado por los contribuyentes para sostener que no existian relaciones contractuales de carácter oneroso, o sea, que la actividad de la sociedad, se realizaba por cuenta de sus accionistas y que, en consecuencia, las cantidades percibidas de éstos, eran simplemente un reintegro de gastos $y$ no retribuciones, por lo que estaban excluidas del impuesto al valor agregado.

La sentencia del Tribunal fue la siguiente:

"De la especial particularidad de la organización ha de extraerse que sin diluir por entero el concepto de personalidades juridicas diferentes (sociedad-accionista-propietario), propio del derecho privado, en el de unidad económica asentada en el derecho tributario, no puede negarse la interdependencia y el alto grado de compenetración que estatutariamente presentan la sociedad $y$ sus accionistas propietarios de unidades. La sociedad es fundamentalmente instrumental. Para tener la condición de accionista, es necesario tener primero la otra, y la explotación de los servicios por cuenta de sus propietarios es elemento prácticamente esencial de la sociedad". 
Asimismo, y nuevamente en la Argentina, en la causa "Kellog" los hechos ocurrían dentro de un conjunto económico entre Kellog de los Estados Unidos (casa matriz) y Kellog S.A. Argentina, fabricante de los bienes que distribuía Kellog Sales sucursal argentina. En esta operación, Kellog S.A. Argentina habia deducido como gastos en sus declaraciones juradas de los años 1970, 1971 y 1982 regalias, servicios técnicos, intereses, diferencias de cambio pagadas a su casa matriz. A su vez, por la fabricación de los productos que distribuía Kellog Sales, sucursal del pais, de la casa matriz, Kellog S.A. le debitó los gastos de fabricación a dicha sucursal más un $4 \%$ como precio de la producción que le entregaba. Entre los gastos debitados a la sucursal, figuraban los correspondientes regalias e intereses que la S.A. pagaba a la casa matriz. La Dirección General Impositiva de Argentina impugnó las deducciones realizadas por concepto de las regalías pagadas a Kellog de los Estados Unidos por la vinculación económica y mantuvo los débitos entre la Sociedad Anónima Argentina y la Sucursal Kellog Sales.

La Sociedad Anónima de Argentina, ante la impugnación de la Dirección General Impositiva, consideró que los réditos generados por su actividad de producción para Kellog Sales (sucursal Argentina) no eran tales ni atribuibles a ella por formar parte de un conjunto económico con la casa matriz al igual que la sucursal argentina.

El Tribunal Fiscal confirmó el criterio de la Dirección General Impositiva. La Corte Suprema lo revocó porque respetó la operación del conjunto económico que en las contrataciones celebradas entre sus integrantes lo fueron como si se tratara de terceros independientes. Así, la doctrina de la penetración (disregard) se deja de lado al no existir actos antijuridicos que importen un abuso del derecho.

De las consideraciones expuestas, puede decirse, en consecuencia, que el principio de la realidad de los hechos económicos instaurado como mecanismo conjuntamente con la interpretación de las normas tributarias, es aquél procedimiento seguido por el intérprete para descubrir el contenido y fin de las normas tributarias, contrastándolos con las formas juridicas adoptadas por los sujetos pasivos en determinadas operaciones comerciales o civiles, debiendo, en la persecución de la verdad $y$, con criterio de justicia, prescindir de tales, tanto a favor del fisco como del contribuyente.

La figura del disregard, se centra en los casos de fraude y, por lo tanto, es más que una forma de interpretar la ley, un criterio adicional de fiscalización para las administraciones tributarias.

\section{La utilización del principio de la primacía de la realidad de los hechos económicos en el Perú}

El primer antecedente encontrado en relación con el tratamiento jurisprudencial del asunto, lo encontramos en el siguiente caso:

En el Perú, desde el 1 de enero de 1943 hasta el 31 de diciembre de 1958, existió el denominado impuesto a las utilidades, para cuya aplicación se consideraba utilidad normal a la que no excediese del $10 \%$ de los recursos sociales o 
patrimonio del contribuyente, reputándose como "sobreutilidad" el exceso de las ganancias sobre dicho porcentaje, exceso sobre el cual, además de abonarse el Impuesto a las Utilidades, se pagaba una tasa adicional del $10 \%$. Para efectuar el cálculo de esta tasa adicional, no se tomaban en cuenta las revaluaciones de activo fijo que efectuase el contribuyente, dado que dichas revaluaciones, al aumentar su patrimonio, incrementarian también el $10 \%$ de dicho patrimonio, o la kutilidad normal" y disminuirian así, con perjuicio del Fisco, la "sobreutilidad".

En el año 1947, un contribuyente, propietario de un fundo lo aportó, revalorizándolo, a la constitución de una sociedad anónima, de la cual poseía la casi totalidad de las acciones. Obviamente, el objeto de todo esto, era disminuir el impuesto a las sobreutilidades a que estaba sujeto. El fisco, al aplicarle el Impuesto a las Utilidades, no le aceptó la revaluación, fundándose en que la nueva sociedad así formada y el fundo que desaparecía como negocio individual, al ser aportado a la sociedad eran patrimonio de un solo y mismo contribuyente, dado que éste era accionista casi exclusivo de la primera y había sido propietario absoluto del fundo, por lo que se podria afirmar que en este caso había una sola unidad económica sobre cuyo patrimonio sin revaluar procedía hacer los cálculos para aplicar el impuestos a las sobreutilidades.

La reclamación que formuló el contribuyente de esta acotación ante la Superintendencia de Contribuciones fue declarada sin lugar, por lo que aquél apeló ante el
Consejo Superior de Contribuciones y obtuvo de éste la Resolución 4328 del 23 de octubre de 1947, que aceptó la revalorización para cómputo de la sobreutilidad de la nueva sociedad así formada. El Superintendente, no satisfecho con esta resolución, y en uso de la facultad que le concedía la Ley 7904 , apeló ante el Supremo Gobierno, el que expidió la Resolución Suprema de 18 de agosto de 1948, que amparó la posición del Fisco.

El contribuyente procedió a demandar la nulidad de tal resolución ante la Corte Suprema, misma que por Ejecutoria del 30 de mayo de 1951, fundada en la doctrina fiscal del 15 del mismo mes y año, dejó establecido que el aportante del fundo y la sociedad que había recibido el aporte, eran contribuyentes distintos.

Mes y medio más tarde, en una apelación de otro contribuyente, el Consejo Superior de Contribuciones, por Resolución 6110 , de 13 de julio de 1951 , revocó la resolución apelada, de acuerdo con el dictamen fiscal del 11 de julio de mismo año, en el que se estableció que el principio de conjunto económico, sostenido por la doctrina, no se encontraba consagrado por la legislación positiva.

Sin embargo a raíz de los importantes cambios acontecidos en la doctrina latinoamericana, el criterio del, en ese tiempo Tribunal Fiscal, cambió ostensiblemente, recogiendo el principio de la primacia de la realidad de los hechos económicos, en sus resoluciones.

Al respecto, Luis Alberto Araoz Villena, en su ponencia "La interpretación económica de las leyes tributarias y la calificación de 
los hechos imponibles por su contenido económico", ha demostrado que los pronunciamientos de la máxima instancia administrativa en materia tributaria han aplicado el principio desde años atrás.

Asi, por ejemplo, se tiene la Resolución 4157 de 12 de diciembre de 1968, en la cual se examinaron dos contratos presentados por la sociedad reclamante; uno de los cuales se había denominado arrendamiento de equipos para fabricación de agua de cola, y el otro, de prestación de servicios por medio del que se proporcionó a otra empresa el personal que operaba los equipos. La reclamante habia pagado por Impuesto a los Timbres el 1\% respecto del primer contrato y e $5 \%$ por el segundo, mientras que la Administración Tributaria había considerado en la acotación que todos los ingresos estaban afectos con la tasa del $5 \%$ por tratarse de una operación única de locación de servicios.

El Tribunal Fiscal consideró que la rescisión de un contrato traia como consecuencia la rescisión del otro, resolviendo que se habia celebrado un solo convenio de prestación de servicios de personal afecto a la tasa del $5 \%$ por Impuesto a los Timbres. Se consideró que el contribuyente había "desdoblado" en dos contratos los que estrictamente era uno, con el objeto de una parte de la retribución estuviera afecta a una tasa menor.

Asimismo, en el caso resuelto por el Tribunal Fiscal 5468 de 3 de agosto de 1970 , se analizaron los contratos de arrendamiento que habian celebrado la reclamante con sus clientes, y que habian sido reparados por la Administración
Tributaria al considerarlos como contratos de compraventa a plazos destinados a disminuir los ingresos por concepto de ventas.

El Tribunal Fiscal confirmó esta apreciación luego de efectuar una revisión de la forma cómo operaba la sociedad, según la cual, si bien extendía los mencionados contratos de arrendamiento, una vez que eran canceladas las letras correspondientes, otorgaba una factura por el total pagado por el cliente, que quedaba como propietario del objeto.

En consecuencia, el Tribunal calificó, al margen de su incorporación en la normativa vigente, el contenido de las operaciones de acuerdo con la realidad de los hechos, cuando las formas jurídicas adoptadas, tenian como finalidad un perjuicio recaudatorio para el Fisco.

\section{La situación actual}

Con la expedición del Decreto Legislativo 816, Código Tributario vigente desde el 22 de abril de 1996, la Norma VIII del mismo consagró en inicio, la siguiente fórmula:

"Al aplicar las normas tributarias podrá usarse todos los métodos de interpretación admitidos por el Derecho.

Cuando los deudores tributarios sometan sus actos a situaciones o relaciones a formas o estructuras jurídicas que no sean manifiestamente las que el derecho privado ofrezca o autorice para configurar la cabal intención económica y efectiva de los deudores tributarios, se prescindirá, en la consideración del hecho imponible real, de las 
formas o estructuras jurídicas adoptadas, y se considerará la situación económica real.

Para determinar la verdadera naturaleza del hecho imponible, la SUNAT tomará en cuenta los actos, situaciones y relaciones económicas que efectivamente realicen, persigan o establezcan los deudores tributarios".

Dicha fórmula, clásica $y$ reiterativa, inspirada en la facción autonomista del derecho tributario, no era sino la expresión normativa del disregard peruano, por cuanto al referirse al alejamiento a las formas que "manifiestamente ofrezca el derecho privado", aludía a la simulación ilícita de los actos jurídicos o el fraude a la ley, sancionado con la inobservancia de los efectos jurídicos requeridos por las partes, tal y como ya se ha definido en las líneas precedentes.

Tal como se ha manifestado, constituye un mecanismo de fiscalización tributaria, pero no una metodologia de interpretación.

Por ello, con la modificatoria introducida por la Ley 26663, a partir del 23 de setiembre de 1996, la Norma VIII suprimió el segundo párrafo, para, en atención a las doctrinas modernas -que incluso han sido ya aceptadas por quienes en un principio sostenian a ultranza la autonomía del derecho tributarioinstaura ya no la teoría del disregard sino, en estricto sentido, el de la primacía de la realidad de los hechos económicos, posición tan defendida en el Uruguay en el cuadragésimo tercer Congreso de la International Fiscal Association de 1989.

En tal sentido, la nueva redacción de la Norma VIII del
Código Tributario, sustancialmente modificada, contiene los siguientes elementos:

a. Se utiliza para determinar la verdadera naturaleza del hecho imponible.

En consecuencia, corresponde a la Administración conocer adecuadamente los elementos del hecho imponible contenidos en la ley, para determinar acertadamente sus alcances y fines y confrontarlos correctamente con las formas jurídicas adoptadas por las partes, de modo tal que no se lleguen a desconocer arbitrariamente.

Esta es pues una norma fundamentalmente programática, orientadora para la Administración, permisora en cuanto a la calificación de los hechos económicos realizados por los deudores tributarios.

b. Tendrá en cuenta los actos, situaciones y relaciones económicas que efectivamente realicen, persigan o establezcan los deudores tributarios.

En tal sentido, el "tener en cuenta" supondrá que la Administración al momento de acercarse a las operaciones realizadas por los contribuyentes, considere el verdadero fin de las mismas, sea a su favor o en su contra.

Luego, se comparten en extenso los principales argumentos previstos por la doctrina y la jurisprudencia rioplatense de 1989 tan bien resumidas por Ramón Valdés Costa en su Curso de Derecho Tributario.

«En nuestro criterio, la solución consiste en respetar equilibradamente los derechos de ambas partes. 
El de los particulares, de adoptar las formas jurídicas más adecuadas a sus necesidades, situación conocida internacionalmente como business purpose, incluso con fines de ahorro impositivo, o con otros fines, como la reorganización de empresas mediante fusiones o separación de sociedades, o la transformación de la naturaleza de éstas, siempre que las nuevas formas no conduzcan a resultados contrarios a la legislación tributaria. El principio aplicable es el de que nadie puede ser privado de lo que la ley no prohibe.

El derecho del Estado a impugnar esas formas, a su vez está limitado a los casos en que éstas afecten la percepción de los tributos, de acuerdo con la ley correctamente interpretada.

La función de la ley es pues, fundamental. Así, se reconoció en el $37^{\circ}$ Congreso de la IFA, al recomendar que los textos legales sean elaborados de manera que la "intención del legislador aparezca claramente enunciada", que "las irregularidades fiscales aparezcan claramente definidas" y al declarar que "la elusión no constituye en esencia, un acto ilegal».

Las discrepancias entre la administración y el contribuyente sobre el alcance de la ley, deben obviamente ser resueltas por los órganos jurisdiccionales, en virtud del principio de la igualdad de las partes.

Si el derecho de los particulares se considera excesivo, o si el texto de la ley no contempla la finalidad perseguida por el legislador al crear el tributo, lo que corresponde es la modificación de la ley. Esa es la tendencia del derecho contemporáneo".
El verdadero significado del principio de primacia de la realidad de los hechos económicos en materia tributaria, ha de constituirse, por lo tanto, en un instrumento que permita al Administrador Tributario reconocer la finalidad del contenido económico de las operaciones realizadas por las partes, el mismo que podrá ser utilizado conjuntamente con los métodos de interpretación aceptados por el derecho.

Se considera, sin embargo, que la fórmula prevista en la Norma VIII del Decreto Legislativo 816, en atención al verdadero sentido del principio de la primacia de la realidad, no debió referirse exclusivamente a la Superintendencia Nacional de Administración Tributaria en particular, sino al "intérprete» en general, con lo cual se incluye a las demás administraciones tributarias, $\mathrm{y}$ al propio contribuyente.

De otro lado, debe entenderse, que la intentio juris de las partes, esto es, los efectos juridicos deseados por las partes en el negocio jurídico realizado, sólo será desatendida por tales administraciones fiscales, en tanto no encuadre con los fines que la norma tributaria persiga, siempre que ella haya sido correctamente interpretada por ellas y sólo cuando se constate que la adopción de determinadas formas juridicas tuvo como objeto el menor pago de los tributos en perjuicio del Estado.

Un desacuerdo entre las partes, esto es, administración y deudor tributario, deberá necesariamente ser resuelto por el Tribunal Fiscal, al que en atención al texto de la ley tributaria y a la modalidad de las operaciones realizadas por el contribuyente, deslindará, al final, 
las verdaderas consecuencias de los hechos económicos así desarrollados por los contribuyentes $\mathrm{y}$ responsables.

Así, esta entidad viene recogiendo esta tendencia tanto a favor del fisco, como ya lo vimos anteriormente, como del contribuyente, pudiendo mencionarse en este último caso los siguientes fallos:

Resolución 4708 de 03 de setiembre de 1969:

"Considerando que de la documentación contable presentada resultaba acreditado que el convenio de industrialización se habia ejecutado a cabalidad, por ambas empresas, y que por tanto no encubría una venta de semillas de algodón, se dejó sin efecto la acotación".

Resolución 18317 de 11 de abril de 1984:

"Teniendo en cuenta que la empresa contabiliza por separado las ventas de bienes y de los diversos servicios, así como los costos y gastos propios de cada actividad, se llega a la conclusión de que se trata de actividades independientes; una industrial de confección por encargo según contrato, que acababa con el producto embolsado; $y$ otra, el servicio de empacado y embalado de mercaderia anteriormente comprada por los terceros a quienes se presta el servicio, de tipo eventual y discontinuo y realizada con materiales proporcionados por ellos, que no forma parte del proceso de fabricación y por lo tanto no gravado con el Impuesto a los Bienes y Servicios".

Como es de apreciarse, el principio de la realidad de los hechos económicos no es una novedad en nuestro sistema jurídico, principio recogido jurisprudencialmente desde mucho atrás y tendencia que luego de la Ley 26663, modificatoria de la Norma VIII del Código Tributario, ha sido plasmada como una recomendación para la Administración, la que al momento de fiscalizar verificará que las formas jurídicas adoptadas por las partes se condiga con la finalidad de las normas tributarias.

\section{CONCLUSIONES}

1. En principio, la interpretación según la realidad económica supone, en buena cuenta, desconocer la eficacia de los efectos jurídicos tributarios si las partes han distorsionado la realidad de una operación económica utilizando una forma jurídica inapropiada que se busca al sólo efecto de defraudar al fisco.

2. En algunas oportunidades, se ha considerado que el principio de la realidad económica es coincidente con el disregard of legal entity o teoría de la penetración.

Sin embargo, sin perjuicio de reconocer ciertos aspectos comunes, se presentan diferencias sustanciales.

Así, se refiere que en común tienen los medios, o sea la facultad del intérprete de desconocer la eficacia de la forma jurídica para adaptarla a la realidad. Es decir, la predominancia de la substancia sobre la forma. 
La diferencia sustancial es, pues, que el disregard se limita expresamente a los casos en que existe fraude o abuso, con lo cual se elimina la posibilidad que el contribuyente puede aplicar el principio de la realidad en su favor, como si procede, por ejemplo, en el caso uruguayo, que sostiene esto como un aspecto esencial de su doctrina.

3. El principio de la realidad de los hechos económicos instaurada como mecanismo de interpretación de las normas tributarias, es aquél procedimiento seguido por el intérprete para descubrir el contenido y el fin de las normas tributarias, contrastándolos con las formas juridicas adoptadas por los sujetos pasivos en determinadas operaciones comerciales o civiles, debiendo, en la persecución de la verdad, y con criterio de justicia, prescindir de tales, tanto a favor del fisco como del contribuyente.

La figura del disregard se centra en los casos de fraude, y por lo tanto, es más que una forma de interpretar la ley, un medio adicional de fiscalización de las administraciones tributarias.

4. El Tribunal Fiscal ha calificado, desde mucho tiempo atrás y al margen de su incorporación en la normativa vigente, el contenido de las operaciones de acuerdo con la realidad económica de los hechos, cuando las formas juridicas adoptadas tenian como finalidad un perjuicio recaudatorio, así como a favor del contribuyente cuando se constataba que tales formas juridicas no se oponian a la intención de las normas tributarias.
5. El verdadero significado del principio de la primacía de la realidad de los hechos económicos en materia tributaria, ha de constituirse por lo tanto, en un instrumento que permita al administrador tributario reconocer la finalidad del contenido económico de las operaciones realizadas por las partes, el mismo que podrá ser utilizado conjuntamente con los métodos de interpretación aceptados por el Derecho.

6. La intentio juris de las partes sólo será desconocida en tanto no encuadre con los fines de la norma tributaria, siempre que ella haya sido correctamente interpretada por el administrador fiscal y cuando se constate que la adopción de determinadas formas jurídicas tuvo como objeto disminuir el pago de los tributos en favor del Estado.

7. Un desacuerdo entre las partes, esto es, administración y deudor tributario, deberá necesariamente ser resuelta por el Tribunal Fiscal, o inclusive por el Poder Judicial, los que interpretando el verdadero sentido de las normas, confrontándolas con las operaciones efectuadas por los contribuyentes, deslindarán las verdaderas consecuencias de los hechos económicos así desarrollados por los deudores tributarios.

8. En tal sentido, la Norma VIII del Código Tributario, sustancialmente modificada, puede ser considerada como un elemento que coadyuve a la Administración Tributaria a determinar el verdadero contenido de las operaciones económicas de los contribuyentes persiguiendo el fin 
de las normas tributarias. Por lo tanto, es una norma fundamentalmente orientadora para la Administración, permisiva en cuanto le permite calificar los hechos económicos efectuados por los deudores tributarios.

Por consiguiente, al intentar buscar la verdad de la norma tributaria, la Administración, al momento de confrontarla con los hechos y las formas juridicas adoptadas por las partes, considerar el verdadero fin de aquélla aún cuando ello no se ajuste a sus intereses fiscales.

\section{BIBLIOGRAFÍA}

1. FOUNROUGE GIULIANI. Derecho Financiero. Vols. I y II. Bs. As. DEPALMA, 1990.

2. INSTTTUTO PERUANO DE DERECHO TRIBUTARIO Jomadas Latinoamericanas de la Internacional Fiscal Association. Publicación efectuada por el .

3. VILLEGAS HÉCTOR Curso de Finanzas, Derecho Financiero y Tributario. Bs. As. DEPALMA, 1990.

4. VALDEZ COSTA, Ramón. Curso de Derecho Tributario. 